\title{
Method for Accurate Quantitation of Volatile Organic Compounds (VOCs) in Urine Using Point of Collection Internal Standard Addition
}

David M. Chambers", Kasey C. Edwards, Eduardo Sanchez, Christopher M. Reese, Alai T. Fernandez, Benjamin C. Blount and Víctor R. De Jesús

Tobacco and Volatiles Branch, Division of Laboratory Sciences, National Center for Environmental Health, Centers for Disease Control and Prevention ${ }^{\dagger}$, Atlanta, GA 30341, USA

* Corresponding author at: Centers for Disease Control, 4770 Buford Highway MS S103-3, Atlanta GA 30341, United States. Tel.: +1 770488 0185; fax: +1 770488 0181. E-mail address: mzz7@cdc.gov

${ }^{\dagger}$ The findings and conclusions in this report are those of the authors and do not necessarily represent the views of the Centers for Disease Control and Prevention. Use of trade names and commercial sources is for identification only and does not constitute endorsement by the U.S. Department of Health and Human Services or the Centers for Disease Control and Prevention.

* Corresponding author at: Centers for Disease Control, 4770 Buford Highway MS S103-3, Atlanta GA 30341, United States. Tel.: +1 770488 0185; fax: +1 770488 0181. E-mail address: mzz7@cdc.gov

${ }^{\dagger}$ The findings and conclusions in this report are those of the authors and do not necessarily represent the views of the Centers for Disease Control and Prevention. Use of trade names and commercial sources is for identification only and does not constitute endorsement by the U.S. Department of Health and Human Services or the Centers for Disease Control and Prevention. 
Supplemental Table 1. Analtye list with corresponding isotopically labeled internal standards (ISTD), limit of detections (LOD), LOD coefficient of variations (CV), and calculated water solubilities and vapor pressures.

\begin{tabular}{|c|c|c|c|c|c|}
\hline Compound & ISTD & $\begin{array}{l}\text { LOD } \\
(\mu \mathrm{g} / \mathrm{L})\end{array}$ & $\begin{array}{l}\% C V \\
\text { at } \\
\text { LOD }\end{array}$ & $\begin{array}{c}\text { Water } \\
\text { Solubility } \\
\text { at } 37^{\circ} \mathrm{C} \\
(\mathrm{mg} / \mathrm{L})^{29}\end{array}$ & $\begin{array}{c}\text { Vapor } \\
\text { Pressure } \\
\text { at } 37^{\circ} \mathrm{C} \\
(\mathrm{Pa})^{29}\end{array}$ \\
\hline Octane & ${ }^{2} \mathrm{H}_{18}$ & 0.050 & 19.7 & 1.57 & 3550 \\
\hline Heptane & ${ }^{2} \mathrm{H}_{16}$ & 0.056 & 21.9 & 6.19 & 10800 \\
\hline$\alpha$-Pinene & ${ }^{2} \mathrm{H}_{3}$ & 0.010 & 11.7 & 6.65 & 1480 \\
\hline Hexane & ${ }^{2} \mathrm{H}_{14}$ & 0.058 & 20.5 & 23.8 & 32700 \\
\hline Isopropylbenzene & ${ }^{2} \mathrm{H}_{5}$ & 0.014 & 15.0 & 90.1 & 1530 \\
\hline Methylcyclopentane & ${ }^{2} \mathrm{H}_{12}$ & 0.019 & 15.9 & 96.9 & 28100 \\
\hline Cyclohexane & ${ }^{2} \mathrm{H}_{12}$ & 0.016 & 16.0 & 113 & 14000 \\
\hline Tetrachloroethylene & ${ }^{13} C_{1}$ & 0.007 & 13.7 & 129 & 7100 \\
\hline 1,2-Dichlorobenzene & ${ }^{13} \mathrm{C}_{6}$ & 0.006 & 20.6 & 186 & 255.7 \\
\hline 1,4-Dichlorobenzene & ${ }^{13} \mathrm{C}_{6}$ & 0.008 & 21.5 & 196 & 349.4 \\
\hline 1,3-Dichlorobenzene & ${ }^{13} \mathrm{C}_{6}$ & 0.003 & 21.6 & 212 & 506.7 \\
\hline Ethylbenzene & ${ }^{13} \mathrm{C}_{6}$ & 0.007 & 21.4 & 246 & 2620 \\
\hline$m$-Xylene & ${ }^{13} \mathrm{C}_{6}$ & 0.015 & 16.6 & 251 & 2010 \\
\hline$p$-Xylene & ${ }^{13} \mathrm{C}_{6}$ & 0.015 & 16.6 & 251 & 2310 \\
\hline o-Xylene & ${ }^{2} \mathrm{H}_{6}$ & 0.009 & 23.1 & 305 & 2170 \\
\hline Styrene & ${ }^{13} \mathrm{C}_{6}$ & 0.017 & 23.8 & 314 & 1360 \\
\hline Trichloroethylene & ${ }^{13} \mathrm{C}_{1}$ & 0.004 & 14.2 & 558 & 25100 \\
\hline Toluene & ${ }^{13} C_{7}$ & 0.013 & 18.7 & 668 & 7100 \\
\hline Chlorobenzene & ${ }^{13} \mathrm{C}_{6}$ & 0.001 & 29.2 & 705 & 2550 \\
\hline Carbon tetrachloride & ${ }^{13} \mathrm{C}_{1}$ & 0.003 & 17.7 & 972 & 24500 \\
\hline 1,1,1,2-Tetrachloroethane & ${ }^{2} \mathrm{H}_{2}$ & 0.004 & 14.0 & 1130 & 3410 \\
\hline$\alpha, \alpha, \alpha$-Trifluorotoluene & ${ }^{2} \mathrm{H}_{5}$ & 0.005 & 12.6 & 1190 & 10200 \\
\hline 1,1,1-Trichloroethane & ${ }^{2} \mathrm{H}_{3}$ & 0.003 & 11.4 & 1270 & 23400 \\
\hline Bromoform & ${ }^{13} \mathrm{C}_{1}$ & 0.005 & 19.5 & 1720 & 1830 \\
\hline Benzene & ${ }^{13} \mathrm{C}_{6}$ & 0.006 & 23.0 & 2040 & 23600 \\
\hline 2,5-Dimethylfuran & ${ }^{13} \mathrm{C}_{2}$ & 0.001 & 22.3 & 2250 & 8070 \\
\hline Nitrobenzene & ${ }^{13} \mathrm{C}_{6}$ & 0.056 & 14.7 & 2370 & 108.5 \\
\hline 1,2,3-Trichloropropane & ${ }^{2} \mathrm{H}_{5}$ & 0.006 & 19.4 & 2790 & 1150 \\
\hline Dibromochloromethane & ${ }^{13} \mathrm{C}_{1}$ & 0.002 & 21.7 & 3380 & 5400 \\
\hline Benzonitrile & ${ }^{2} \mathrm{H}_{5}$ & 0.056 & 16.7 & 4360 & 214.2 \\
\hline Chloroethane & ${ }^{2} \mathrm{H}_{5}$ & 0.031 & 25.7 & 5020 & 222000 \\
\hline Vinyl bromide & ${ }^{2} \mathrm{H}_{3}$ & 0.006 & 14.8 & 5310 & 155000 \\
\hline Bromodichloromethane & ${ }^{13} \mathrm{C}_{1}$ & 0.002 & 19.6 & 6310 & 16000 \\
\hline 1,2-Dibromoethane & ${ }^{13} \mathrm{C}_{2}$ & 0.003 & 19.1 & 8370 & 2500 \\
\hline 2-Hexanone & ${ }^{2} \mathrm{H}_{5}$ & 0.025 & 11.9 & 8430 & 3310 \\
\hline Chloroform & ${ }^{13} C_{1}$ & 0.003 & 17.4 & 10800 & 41000 \\
\hline 1,2-Dichloroethane & ${ }^{2} \mathrm{H}_{4}$ & 0.003 & 17.0 & 12100 & 24400 \\
\hline Furan & ${ }^{2} \mathrm{H}_{4}$ & 0.006 & 15.0 & 12800 & 85300 \\
\hline Methyl isobutyl ketone & ${ }^{13} \mathrm{C}_{6}$ & 0.008 & 22.2 & 13000 & 56200 \\
\hline Methyl tert-butyl ether & ${ }^{2} \mathrm{H}_{12}$ & 0.007 & 20.3 & 13000 & 4220 \\
\hline Ethyl acetate & ${ }^{2} \mathrm{H}_{8}$ & 0.099 & 19.2 & 28300 & 18500 \\
\hline Ethyl ether & ${ }^{2} \mathrm{H}_{10}$ & 0.013 & 13.3 & 35400 & 77800 \\
\hline Methylene chloride & ${ }^{13} \mathrm{C}_{1}$ & 0.016 & 45.0 & 44200 & 83100 \\
\hline Isobutyronitrile & ${ }^{2} \mathrm{H}_{6}$ & 0.014 & 22.9 & 116000 & 16100 \\
\hline Tetrahydrofuran & ${ }^{2} \mathrm{H}_{8}$ & 0.050 & 23.2 & 201000 & 32200 \\
\hline
\end{tabular}

